\title{
Wuzhi Tablet (Schisandra sphenanthera Extract) Is a Promising Tacrolimus-Sparing Agent for Renal Transplant Recipients Who Are CYP3A5 Expressers: a Two-Phase Prospective Study
}

\author{
Jiali Li, Siyang Chen, Xiaoling Qin, Qian Fu, Huichang Bi, Yu Zhang, Xueding Wang, Longshan Liu, \\ Changxi Wang, and Min Huang
}

Institute of Clinical Pharmacology, School of Pharmaceutical Sciences, Sun Yat-sen University, Guangzhou, China (J.L., X.Q., H.B., Y.Z., X.W., M.H.); Kidney Transplant Department, Transplant Center, The First Affiliated Hospital of Sun Yat-sen University, Guangzhou, China (S.C., Q.F., L.L., C.W.); Guangdong Food and Drug Vocational College, Guangzhou, China (X.Q.); and School of Pharmaceutical Sciences, Guangzhou Medical University, Guangzhou, China (Y.Z.)

Received May 15, 2017; accepted August 17, 2017

\section{ABSTRACT}

Tacrolimus is a potent but expensive first-line immunosuppressant, thus solutions to reduce tacrolimus consumption while maintain therapeutic level are in urgent need. A two-phase prospective study was conducted to assess the efficacy of an ethanolic extraction preparation of Schisandra sphenanthera (Wuzhi tablet) as a tacrolimus-sparing agent in renal transplant recipients who were high-dose tacrolimus consumers (CYP3A5*1 allele carriers, CYP3A5 expressers). A total of 12 patients were included in the Part I study. After co-administration of Wuzhi tablet, the average individual increment $(\%)$ in dose-adjusted $\mathrm{C}_{0}, \mathrm{C}_{\max }$ and AUC $_{0-12}$ hour of tacrolimus were $198.8 \%(95 \% \mathrm{Cl} 149.2,248.3)$, $111.0 \%(95 \% \mathrm{Cl} 63.4,158.6)$ and $126.1 \%(95 \% \mathrm{Cl} 89.4,162.8)$, respectively $(P<0.01)$, while the average individual reduction $(\%)$ in tacrolimus daily dose was $40.9 \%(95 \%$ Cl $25.2,56.6)(P<0.01)$.
Subsequently, 32 patients were enrolled in a prospective, randomized, controlled study and randomly assigned to receive tacrolimus by CYP3A5 genotype plus Wuzhi tablet co-administration guided dosing (study group) or standard dosing (control group). Besides less tacrolimus dose requirement $(P<0.01)$, a more accurate tacrolimus initial dose characterized by lower incidence of out-ofrange $C_{0}$ after initial dose $(P<0.01)$ and fewer dose changes $(P<$ 0.01 ) was found in the study group. Moreover, no significant differences in acute rejection rate and serum creatinine levels were observed between two groups. Our results show that CYP3A5 genotype plus Wuzhi tablet co-administration guided tacrolimus dosing is a promising therapy for CYP3A5 expressers in the early post-transplant stage, while further study with a larger sample size is required to prove these findings.
Introduction

Tacrolimus (FK506), a macrolide isolated from the fermentation broth of Streptomyces tsukubaensis (Montini et al., 2006), is the first-line immunosuppressant used for the prevention of allograft rejection after solid organ transplantation. However, as an expensive drug, almost entire post-transplant life-long treatment with tacrolimus imposes a substantial financial burden on both patients and healthcare insurance systems. Therefore, during these years, both clinicians and pharmaceutical researchers have been trying to solve a problem, that is, how to

The authors appreciate the financial supports provided by the National Major Projects for science and technology development from Science and Technology Ministry of China [Grant 2012ZX09506001-004]; National Natural Science Foundations of China [Grants 81102515, 81320108027]; Natural Science Foundations of Guangdong Province, China [Grant 2016A030313219]; National Key Research and Development Program [Grant 2016YFC0905000]; the 111 Project [Grant B16047]; Key Laboratory Foundation of Guangdong Province [Grant 2011A060901014] and Major Scientific and Technological Project of Guangdong Province [Grants 2011A080300001, 2012A080202013).

https://doi.org/10.1124/dmd.117.076737. reduce tacrolimus consumption while maintain its therapeutic level (Jones and Morris, 2002; El-Dahshan et al., 2006; Liu et al., 2009).

Tacrolimus is predominantly metabolized in the liver and in the small intestine by cytochrome P450 3A (including CYP3A4 and CYP3A5), and its absorption is modulated through P-glycoprotein (P-gp) (Masuda and Inui, 2006). Thus, co-administration with inhibitors of CYP3A and/or P-gp to increase the blood level of tacrolimus, has been demonstrated to be an effective way for decreasing tacrolimus consumption (Hebert and Lam, 1999; Jones and Morris, 2002; El-Dahshan et al., 2006). Calcium channel blocker diltiazem, an inhibitor of both CYP3A4/5(Jones et al., 1999) and P-gp(Cornwell et al., 1987), has been recommended as a tacrolimus-sparing agent by Kidney Disease: Improving Global Outcome (KDIGO) clinical practice guideline(Kidney Disease: Improving Global Outcomes Transplant Work, 2009).

Wuzhi tablet (registration number in China: Z20025766), an ethanolic extraction preparation of Schisandra sphenanthera (also called "wuwei-zi" in China), is widely used for the treatment of viral and druginduced hepatitis in China (Loo et al., 2007). Ethanolic extract of per gram of Wuzhi tablet powder contains $7.244 \pm 0.777 \mathrm{mg}$ Schisandrin A, $0.017 \pm 0.001 \mathrm{mg}$ Schisandrin B, $0.024 \pm 0.003 \mathrm{mg}$ Schisandrin C, $0.048 \pm 0.002 \mathrm{mg}$ Schisandrol A, $0.468 \pm 0.020 \mathrm{mg}$ Schisandrol B and

ABBREVIATIONS: $\mathrm{AUC}_{0-12} \mathrm{~h}$, area under the concentration-time curve from 0 to $12 \mathrm{~h}$; $\mathrm{C}_{\max }$, peak concentration; $\mathrm{C}_{0}$, trough concentration; LC-MS/MS, liquid chromatography-tandem mass spectrometry; PCR-RFLP, polymerase chain reaction- restriction fragment length polymorphism; P-gp, P-glycoprotein; TDM, therapeutic drug monitoring; t.i.d., ter in die; $t_{\max }$, time to peak concentration. 
$10.089 \pm 1.221 \mathrm{mg}$ Schisantherin A, which was determined by a validated liquid chromatography-tandem mass spectrometry (LCMS/MS) analysis developed in our laboratory (Qin et al., 2013). Among these dibenzocyclooctene lignans, Schisandrin A, Schisandrin B, Schisandrol A, Schisandrol B and Schisantherin A were shown to be P-gp inhibitors(Pan et al., 2006; Wan et al., 2006; Fong et al., 2007), while Schisandrin A, Schisandrin B and Schisantherin A were reported to potently inhibit CYP3A4 (Iwata et al., 2004). Therefore, it is likely that Wuzhi tablet will interact with the substrates of CYP3A and/or P-gp, and suggesting that Wuzhi tablet could enhance the bioavailability of tacrolimus and used as a sparing agent.

Therefore, we conducted a two-phase prospective study to assess the efficacy of Wuzhi tablet as a tacrolimus-sparing agent in renal transplant recipients who were high-dose tacrolimus consumers. $C Y P 3 A 5^{*} 1$ allele carriers (carriers of $C Y P 3 A 5 * 1 / * 1$ genotype or $C Y P 3 A 5 * 1 / * 3$ genotype, CYP3A5 expressers), who have been proved to be associated with lower tacrolimus exposure by a number of reports including our previous studies (Li et al., 2011, 2015; Kurzawski et al., 2014), were enrolled in this study. Firstly, we investigated the tacrolimus-sparing effect of Wuzhi tablet by a pharmacokinetic study in which each patient served as his or her own control. Secondly, based on the tacrolimus-sparing effect of Wuzhi tablet, an individualized tacrolimus-sparing dosing regimen guided by CYP3A5 genotype and Wuzhi tablet co-administration was established and used to guide tacrolimus initial dosing in renal transplant recipients by a prospective, randomized, controlled study.

\section{Materials and Methods}

Study Design and Patients. This work was performed in accordance with the current Declaration of Helsinki and Good Clinical Practice guidelines. The protocol was approved by the Ethics Committee of the First Affiliated Hospital of Sun Yat-sen University.

The study consisted of two prospective studies. In the first part (Part I), adult (18-60 years) male and female patients receiving single primary living-donors renal transplantation in Kidney Transplant Department, the First Affiliated Hospital of Sun Yat-Sen University (KTD-SYSU) between June 2012 and October 2013 were eligible. The patients initiated tacrolimus-based triple immunosuppressive regimen according to the routine protocol of KTD-SYSU (Li et al., 2011). Specifically, tacrolimus (Prograft; Astellas, Killorglin, Ireland) was given $0.05-0.075 \mathrm{mg} / \mathrm{kg}$ twice daily, mycophenolate mofetil (Cellcept; Roche, Basel, Switzerland) was taken 1.0-1.5 g/d and prednisone (Guangdong Huanan Pharmacy Ltd., Dongguan, China) was given $30 \mathrm{mg} / \mathrm{d}$. Those with abnormal hepatic function or receiving combined organ transplantations were excluded. The absence of medication known to affect tacrolimus blood levels (except for prednisone), such as diltiazem, verapamil, rifampicin, ketoconazole, itraconazole, erythromycin, clarithromycin or phenytoin, were checked for each patient. Patients were genotyped for CYP3A5*3 (6986A > G) after renal transplantation. A total of twelve CYP3A5 expressers (10 males and 2 females; mean age: $31.4 \pm 11.0$ years with a range of $18-53$ years; one patient carried CYP $3 A 5 * 1 / * 1$ genotype, and the others were CYP $3 A 5 * 1 / * 3$ genotype carriers), who had took tacrolimus for 2 weeks and stable concentration level has reached, were enrolled and given Wuzhi tablet $(0.62$ g, t.i.d.; Guangxi Fanglue Pharmaceutical Co., Ltd., Nanning, China) for 14 days in addition to their normal triple immunosuppressive regimen. Tacrolimus pharmacokinetics was measured on the day before initiation of Wuzhi tablet and on day 14 of Wuzhi tablet supplement. Whole blood samples were taken before and at $0.5,1,1.5,2,3,4,6$, 9 and 12 hours after tacrolimus administration. Trough levels $\left(\mathrm{C}_{0}\right)$ of tacrolimus were monitored every 3 days and dosages were subsequently adjusted to achieve therapeutic concentration between 5 and $10 \mathrm{ng} / \mathrm{ml}$.

In the second part (Part II), 32 CYP3A5 expressers were recruited before their primary living-donor renal transplantation in KTD-SYSU between June 2014 and June 2016. They were randomly and evenly assigned to "control" or "study" group. In control group, patients received routine initial tacrolimus-based triple immunosuppressive regimen as described in Part I study, without co-administration of Wuzhi tablet. In study group, Wuzhi tablet $(0.62 \mathrm{~g}$, t.i.d. $)$ was co-administered, initial dose of tacrolimus was calculated basing on the
CYP3A5 genotype-guided algorithm developed from our previous study (Li et al., 2011) and the tacrolimus-sparing effect of Wuzhi tablet (results of Part I), while other immunosuppresants used were in accordance to the routine protocol of KTD-SYSU. The exclusion criteria were the same as those in Part I. The primary end points were the accuracy of the initial dose and the dose requirement of tacrolimus, including: 1 ) the $\mathrm{C}_{0}$ after the initial dose, which was determined 3 days after the initiation of tacrolimus when steady-state concentration has been achieved, 2) the proportion of out-of-range $\mathrm{C}_{0}$ (defined as $\mathrm{C}_{0}<5$ or $\mathrm{C}_{0}>$ $10 \mathrm{ng} / \mathrm{ml}$ ) after initial dose, 3) the number of dose adjustments made to achieve therapeutic $\mathrm{C}_{0}$ range of $5-10 \mathrm{ng} / \mathrm{ml}$, and 4) the dose requirement to reach therapeutic $\mathrm{C}_{0}$ range. The secondary endpoints included incidences of biopsyproved acute rejection within 2 weeks after transplantation and renal allograft function represented by serum creatinine on 14 days after transplantation.

Quantitation of Tacrolimus in Whole Blood. Whole blood concentrations of tacrolimus were determined by our previously developed liquid chromatography-tandem mass spectrometry (LC-MS/MS) (Li et al., 2008).

Pharmacokinetic Calculations. Trough concentration $\left(\mathrm{C}_{0}\right)$, peak concentration $\left(\mathrm{C}_{\max }\right)$ and time to peak concentration $\left(\mathrm{t}_{\max }\right)$ of tacrolimus were read directly from the observed whole blood concentration versus time profiles. The area under the whole blood concentration versus time from 0 to 12 hour $\left(\mathrm{AUC}_{0-12}\right.$ hour $)$ of tacrolimus was calculated using the linear trapezoidal rule by DRUG AND STATISTICS (DAS) software (version 2.1.1; Drug and Statistics, Mathematical Pharmacology Professional Committee of China). Correlations between $\mathrm{C}_{0}$ with the $\mathrm{AUC}_{0-12}$ hour before and after co-administration of Wuzhi tablet were assessed.

DNA Extraction and Genotyping. Total genomic DNA was extracted from peripheral blood leukocytes according to the sodium iodide method described previously (Loparev et al., 1991). The CYP3A5*3 (6986 A > G) polymorphism was genotyped by using a previously published polymerase chain reactionrestriction fragment length polymorphism (PCR-RFLP) method (Zhang et al., 2013).

Statistical Analysis. Statistical analyses were performed in SPSS (Statistical Package for the Social Sciences) software (version 21; SPSS, IBM, NY). The data are expressed as mean \pm S.D. The comparison of pharmacokinetic before and after co-administration of Wuzhi tablet was conducted using a Wilcoxon SignedRank Test. Mann-Whitney $U$ test or $\chi^{2}$ test was used for comparisons between continuous variables or discrete variables, respectively. Spearman's correlation was used to evaluate the correlation between $\mathrm{C}_{0}$ and $\mathrm{AUC}_{0-12 \text { hour }}$.

A $P$-value less than 0.05 was considered as statistically significant. Statistical power of the sample size was calculated by using PASS (Power Analysis and Sample Size) software (version 11.0.7, PASS; NCSS, LLC, Atlanta, GA). All the results met the requirement to have more than $80 \%$ power to detect the difference between/among groups with two-side type one error at $5 \%$.

\section{Results}

Part I. The mean whole blood concentration-time curve of tacrolimus before and after co-administration of Wuzhi tablet in 12 renal transplant recipients was shown in Fig. 1. Pharmacokinetics of tacrolimus before and after co-administration of Wuzhi tablet in 12 renal transplant recipients was presented in Table 1 . Individual data on tacrolimus doseadjusted $\mathrm{C}_{0}, \mathrm{C}_{\max }$ and $\mathrm{AUC}_{0-12}$ hour before and after co-administration of Wuzhi tablet were shown in Fig. 2.

After co-administration of Wuzhi tablet, the average individual increment (\%) in dose-adjusted $\mathrm{C}_{0}, \mathrm{C}_{\max }$ and $\mathrm{AUC}_{0-12}$ hour of tacrolimus were $198.8 \%$ (95\% CI 149.2, 248.3), $111.0 \%$ (95\% CI 63.4, 158.6) and $126.1 \%$ (95\% CI 89.4, 162.8), respectively $(P<0.01)$. The time to peak concentration $\left(\mathrm{t}_{\max }\right)$ was significantly prolonged $(2.4 \pm 0.9$ hour vs. 1.7 \pm 1.0 hour, $P=0.034)$. The average individual reduction $(\%)$ in tacrolimus daily dose was $40.9 \%(95 \%$ CI $25.2,56.6)(P<0.01)$. Correlation between $\mathrm{C}_{0}$ and $\mathrm{AUC}_{0-12}$ hour were: 1) before co-administration of Wuzhi tablet, $r=0.872(P<0.01)$, and 2$)$ after co-administration of Wuzhi tablet, $r=0.921(P<0.01)$.

Part II. As shown in Table 2, the distributions of age, gender and CYP3A5 genotype were balanced in the control and study groups.

According to the genotype-guided algorithm: $\mathrm{C}_{0} /$ Dose $=49.226 \times$ CYP3A5 + 76.053, in which CYP3A5 nonexpressers was coded as 1 , 


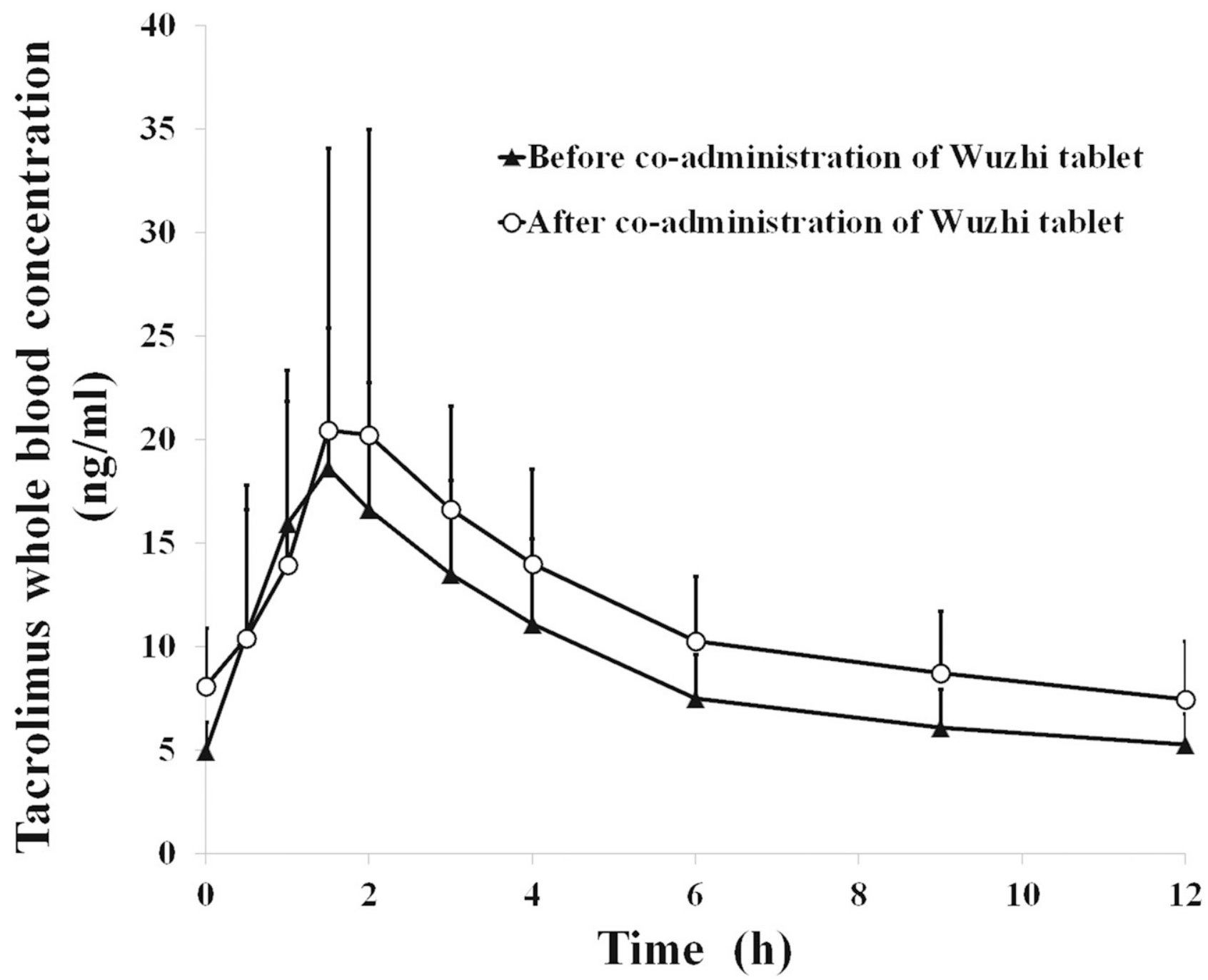

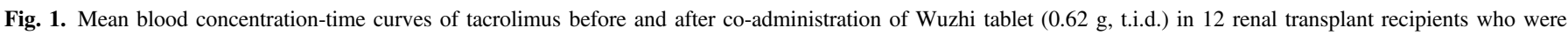
CYP3A5 expressers.

CYP3A5 expressers was coded as 0 (Li et al., 2011). With targeting $\mathrm{C}_{0}$ at $8 \mathrm{ng} / \mathrm{ml}$, the calculated initial single dose was about $0.105 \mathrm{mg} / \mathrm{kg}$ in CYP3A5 expressers. From the results of Part I, co-administration of Wuzhi tablet could result in 2.26-fold increase in the dose-adjusted $\mathrm{AUC}_{0-12 \text { hour }}$ of tacrolimus, we speculated that to achieve the same level of drug exposure, patients co-administered with Wuzhi tablet should require about $55 \%$ less tacrolimus than those without Wuzhi tablet co-administration. Therefore, the initial single dose of tacrolimus in the study group was set at $0.05 \mathrm{mg} / \mathrm{kg}$.

Compared with the control group, the average initial tacrolimus single dose was $21.9 \%$ lower $(0.050 \pm 0.002 \mathrm{mg} / \mathrm{kg}$ vs. $0.064 \pm$
$0.006 \mathrm{mg} / \mathrm{kg}, P<0.01$ ) while the average $\mathrm{C}_{0}$ of tacrolimus after initial dose was $70.4 \%$ higher $(7.31 \pm 2.27 \mathrm{ng} / \mathrm{ml}$ vs. $4.29 \pm$ $1.42 \mathrm{ng} / \mathrm{ml}, P<0.01)$ in the study group. The proportion of out-ofrange $\mathrm{C}_{0}$ of tacrolimus after initial dose was significantly dropped from $68.8 \%$ to $12.5 \%(P<0.01)$, and accordingly the dose adjustments were markedly decreased from $1.33 \pm 0.78$ times to $0.17 \pm 0.39$ times $(P<0.01)$ in the study group. A reduction of $37.5 \%$ in tacrolimus dose requirement $(0.050 \pm 0.006 \mathrm{mg} / \mathrm{kg}$ vs. $0.080 \pm 0.012 \mathrm{mg} / \mathrm{kg}, P<$ $0.01)$ to reach therapeutic range was observed in study group in comparison with that in control group. The incidences of biopsyproved acute rejection within 2 weeks after transplantation and serum

TABLE 1

Pharmacokinetic of tacrolimus before and after co-administration of Wuzhi tablet $(0.62 \mathrm{~g}$, t.i.d.) in 12 renal transplant recipients who were CYP3A5 expressers

\begin{tabular}{lcccc}
\hline & $\mathrm{C}_{0} /$ Dose $(\mathrm{ng} / \mathrm{ml}) /\left(\mathrm{mg}^{\prime} \mathrm{kg}\right)$ & $\mathrm{C}_{\max } /$ Dose $(\mathrm{ng} / \mathrm{ml}) /(\mathrm{mg} / \mathrm{kg})$ & $\mathrm{AUC}_{0-12 \mathrm{~h}} / \mathrm{Dose}(\mathrm{ng} / \mathrm{ml} \cdot \mathrm{h}) /(\mathrm{mg} / \mathrm{kg})$ & $\mathrm{t}_{\max }(\mathrm{h})$ \\
\hline Before & $96.9 \pm 46.5$ & $382.3 \pm 192.2$ & $2328.0 \pm 1356.6$ & $1.7 \pm 1.0$ \\
After & $278.5 \pm 119.2^{* *}$ & $809.2 \pm 525.1^{* *}$ & $4892.4 \pm 2173.1^{* *}$ & $2.4 \pm 0.9^{*}$ \\
\hline
\end{tabular}

$\mathrm{AUC}_{0-12 \mathrm{~h}}$, area under the concentration-time curve from 0 to $12 \mathrm{~h} ; \mathrm{t}_{\max }$, time to peak concentration $\mathrm{C}_{\max }$, peak concentration; $\mathrm{C}_{0}$, trough concentration.

$* P<0.05 ; * * P<0.01$ for comparison between before and after co-administration of Wuzhi tablet. 
A

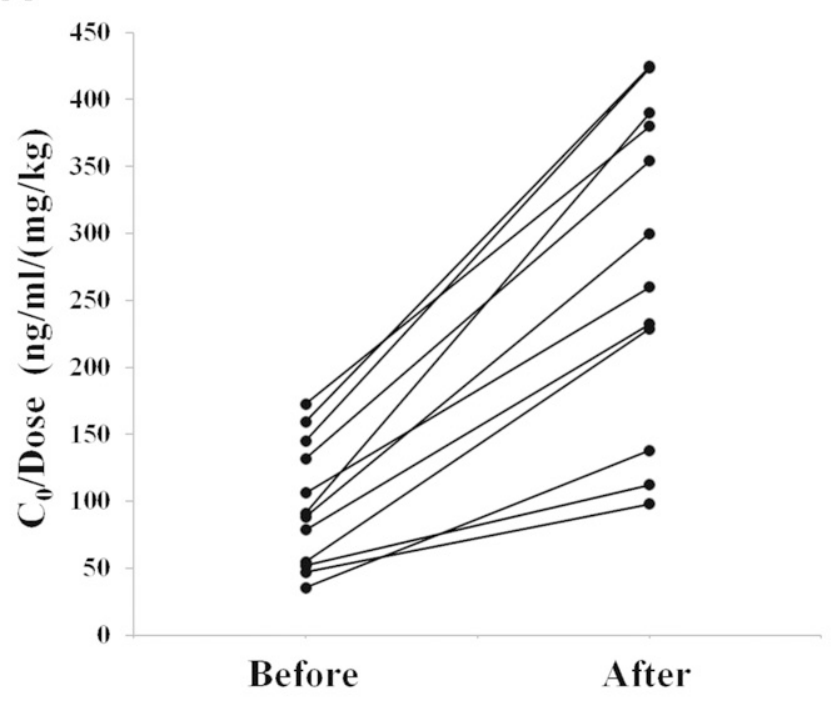

C

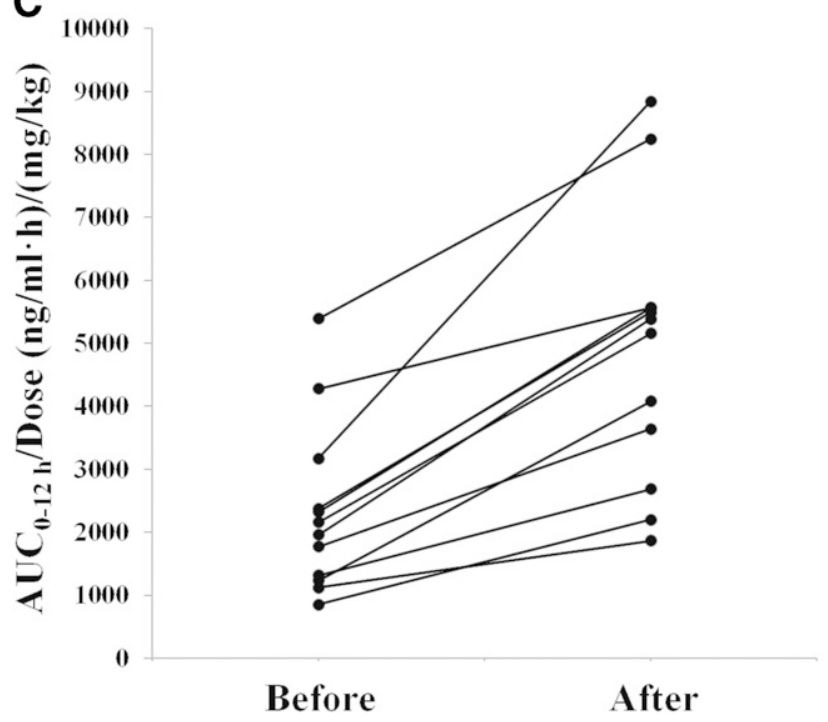

B

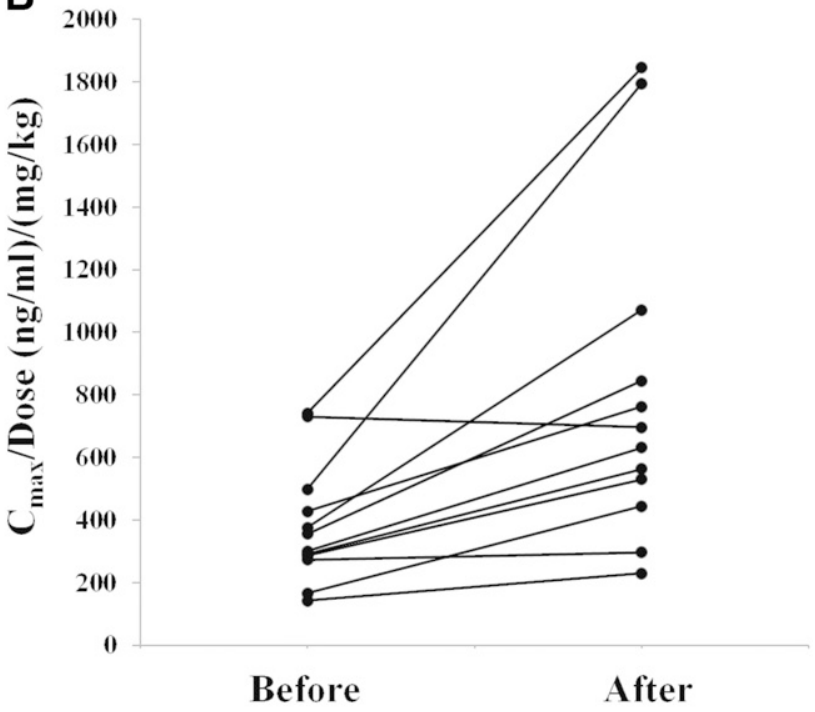

D

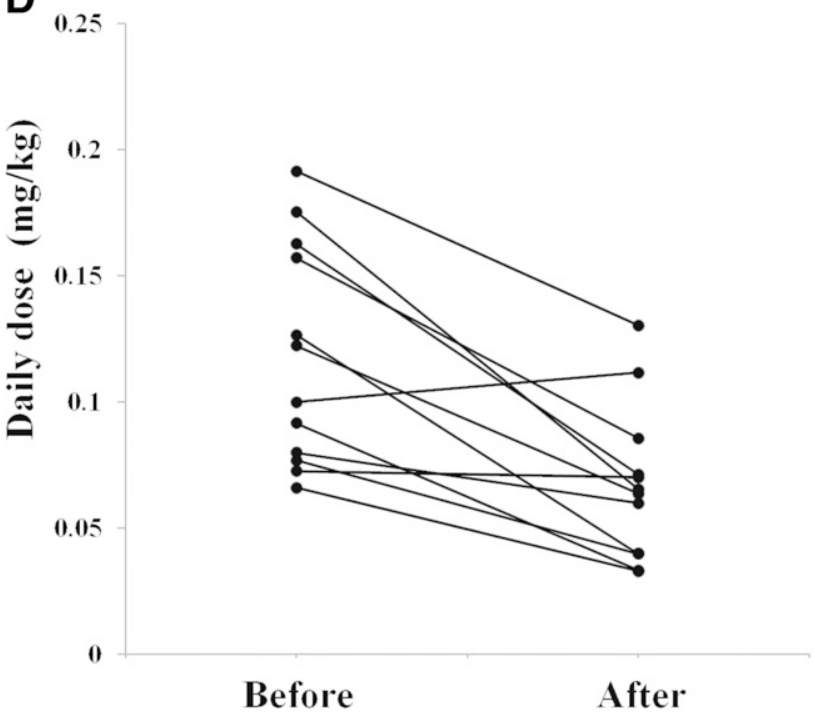

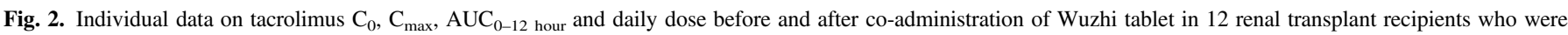

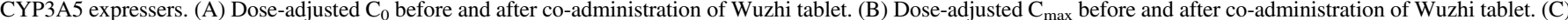

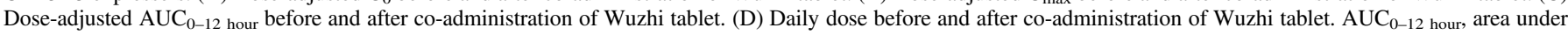
the concentration-time curve from 0 to 12 hour; $\mathrm{C}_{\max }$, peak concentration; $\mathrm{C}_{0}$, trough concentration.

creatinine on 14 days after transplantation were not different between two groups.

\section{Discussion}

The bioavailability of tacrolimus is primarily determined by CYP3A activity in the gut and liver and P-gp activity in the gut. Drug-drug interactions between tacrolimus and inducers or inhibitors of CYP3A and/or P-gp have been extensively reported, including diltiazem (Hebert and Lam, 1999; Jones and Morris, 2002) and rifampicin (Chenhsu et al., 2000), etc. With the wide spread use of herb drug, the herb-drug interactions of tacrolimus with herb drugs have become more and more common. St. John's Wort (Hypericum perforatum) was found to significantly enhance tacrolimus metabolism, through induction of both CYP3A and P-gp (Hebert et al., 2004). To the contrary, grapefruit juice could markedly increase bioavailability of tacrolimus with its active components potently inhibiting CYP3A4 (Liu et al., 2009). In the present study, we conducted a two-phase prospective study to investigate the influences of an ethanolic extraction preparation of Schisandra sphenanthera (Wuzhi tablet) on the pharmacokinetics and dose requirement of tacrolimus in renal transplant recipients carrying CYP $3 A 5 * 1$ allele. According to the increment of dose-adjusted $\mathrm{AUC}_{0-12}$ hour of tacrolimus after co-administration of Wuzhi tablet from Part I study, we speculated that to achieve the same level of drug exposure, CYP3A5 expressers co-administered with Wuzhi tablet should require about $55 \%$ less tacrolimus than those without Wuzhi tablet co-administration. In the subsequent Part II study, when achieving the therapeutic range, a $37.5 \%$ decline in tacrolimus dose requirement was observed in the study group in comparison with that in control group, which was a little lower than our expectation. However, the mean $\mathrm{C}_{0}$ was approximately $13 \%$ higher in the study group than that in the control group when achieving the therapeutic range $(7.26 \pm 1.84 \mathrm{ng} / \mathrm{ml} \mathrm{vs} .6 .42$ $\pm 1.00 \mathrm{ng} / \mathrm{ml}$ ), suggesting that the dose requirement of tacrolimus in the study group could be further reduced by about $11.5 \%$ to obtain the same 
TABLE 2

Patient's demographic details, primary and secondary end points in the Part II study

\begin{tabular}{lcc}
\hline & Control group $(n=16)$ & Study group $(n=16)$ \\
\hline Gender (male/female) & $10 / 6$ & $11 / 5$ \\
Age $(\mathrm{yr})$ & $37.7 \pm 10.3$ & $39.3 \pm 12.9$ \\
CYP3A5 genotype & 2 & 4 \\
$C Y P 3 A 5^{*} 1 / * 1$ & 14 & 12 \\
CYP3A5*1/*3 & $0.064 \pm 0.006$ & $0.050 \pm 0.002^{* *}$ \\
Initial tacrolimus single dose $(\mathrm{mg} / \mathrm{kg})$ & $4.29 \pm 1.42$ & $7.31 \pm 2.27 * *$ \\
$\mathrm{C}_{0}$ of tacrolimus after initial dose $(\mathrm{ng} / \mathrm{ml})$ & $68.6 \%$ & $12.5 \% * *$ \\
Percent of out-of-range $\mathrm{C}_{0}$ after initial dose & $1.33 \pm 0.78$ & $0.17 \pm 0.39^{* *}$ \\
Number of dose adjustment to reach therapeutic range & $0.080 \pm 0.012$ & $0.050 \pm 0.006^{* *}$ \\
Dose needed to achieve therapeutic range $(\mathrm{mg} / \mathrm{kg})$ & $6.25 \%$ & $6.25 \%$ \\
Acute rejection rates within 2 wk after transplantation & $118.4 \pm 29.1$ & $124.3 \pm 30.5$ \\
Serum creatinine on day 14 after transplantation $(\mu \mathrm{mol} / \mathrm{l})$ & & \\
\hline $\mathrm{C}_{0}$, trough concentration. & &
\end{tabular}

$\mathrm{C}_{0}$ as that in the control group. These results indicated that Wuzhi tablet could be used as a tacrolimus-sparing agent.

Previous in vitro and in vivo experiments in our laboratories have elucidated the direct inhibitory effect of Wuzhi tablet on the activity of P-gp and CYP3A4, by using digoxin and midazolam (typical P-gp and CYP3A substrates) as probe substrates (Xue et al., 2013). Similarly, we demonstrated that Wuzhi tablet could significantly enhance the bioavailability of tacrolimus primarily through the inhibition of P-gpmediated efflux and CYP3A-mediated metabolism in the intestine (Qin et al., 2010b). We further clearly clarified the involved mechanisms in the interaction between tacrolimus and the six major bioactive lignans in Wuzhi tablet, indicating that Schisandrol B was the most potent component contributing to the enhancement of the AUC, the oral bioavailability, the gut processes affecting availability, and the hepatic availability of tacrolimus (Qin et al., 2014).

In our previous study in rats, the $\mathrm{AUC}_{0-\infty}$ value of tacrolimus was increased by 3.1 fold after co-administration of Wuzhi tablet at dosage extrapolated from the clinical practice in treating viral and drug-induced hepatitis (0.93 g, t.i.d.) (Qin et al., 2010b). Moreover, the inhibitory effect of Wuzhi tablet or the six lignans on tacrolimus metabolism was observed to be in a dose-dependent manner (Qin et al., 2014). Therefore, to avoid a sharp rise in tacrolimus concentration, the dosage of Wuzhi tablet used here was lower than that recommended for treating viral and drug-induced hepatitis. Moreover, tacrolimus trough concentrations were closely monitored and dosages were adjusted to achieve therapeutic concentration.

Several studies have indicated that tacrolimus $\mathrm{C}_{0}$ was closely associated with the risk of rejection and toxicity during the crucial first week after transplantation when the patients were most likely to be vulnerable to early organ rejection (Laskow et al., 1996; Aidong et al., 2004). Therefore, the importance of prescribing the most appropriate dose of tacrolimus and achieving therapeutic trough concentration timely during the initial post-transplant period becomes clinically valuable. In the current study, under the CYP3A5 genotype plus Wuzhi tablet combination guided tacrolimus dosing regimen, the accuracy of tacrolimus initial dose for CYP3A5 expressers was significantly improved characterized by promising reductions in out-of-range $\mathrm{C}_{0}$ after initial dose and dose changes, indicating that CYP3A5 expressers in study group may reach tacrolimus therapeutic level more promptly than those in control group.

Furthermore, acute rejection rate and serum creatinine levels in the study group was comparable to that in the control group, indicating that the immunosuppressive effect of tacrolimus or renal allograft function were not impaired by the supplement of Wuzhi tablet during the study period. Additionally, our previous studies in rats indicated that Wuzhi tablet potently increased tacrolimus oral bioavailability but not significantly influenced its tissue distribution (Qin et al., 2010a). Besides, drug-induced hepatitis is common in renal transplant recipients because many other kinds of drugs (e.g., anti-infectives, cardiovascular drugs, etc.) with hepatotoxicity are often co-administrated to prevent posttransplant side effects. These findings implied that Wuzhi tablet, also a hepatoprotective, may be a safe and promising tacrolimus-sparing agent.

Tacrolimus $\mathrm{C}_{0}$ levels are commonly used for routine therapeutic drug monitoring (TDM) to guide concentration-controlled dosing, since they have been found to correlate well with AUC (Jusko et al., 1995; Venkataramanan et al., 1995). Whether such a good correlation would be influenced by the supplement of Wuzhi tablet was also taken into our consideration. An even better correlation was seen after co-administration of Wuzhi tablet compared with that before co-administration ( $r=0.921$ vs. 0.872 ), suggesting that $\mathrm{C}_{0}$ is still an ideal drug exposure marker of tacrolimus when Wuzhi tablet is co-administered.

The influences of the extract of Schisandra sphenanthera on tacrolimus pharmacokinetics have been reported in healthy volunteers and liver transplant recipients(Xin et al., 2007; Jiang et al., 2010), while for renal transplant recipients, only the differences in $\mathrm{C}_{0}$ between the groups with and without co-administration of the extract of Schisandra sphenanthera were investigated by two case-control studies(Xin et al., 2011; Wang et al., 2016). However, another preparation of extract of Schisandra sphenanthera called Wuzhi capsule was used in these studies, and the quality control constituent in Wuzhi capsule is Schisandrin A while in Wuzhi tablet is Schisantherin A probably due to the different pharmaceutical technologies. The content of major active constituents in Wuzhi tablet has been quantified and the underlying mechanism in the interaction between tacrolimus and the major bioactive constituents has been clearly clarified in our laboratory (Qin et al., 2013). Most importantly, an individualized tacrolimus dosing regimen by taking both of $C Y P 3 A 5$ genotype guided algorithm and the tacrolimus-sparing effect of Wuzhi tablet into consideration, has been successfully applied to guide individualized tacrolimus dosing in renal recipients, which has never been reported elsewhere.

The findings in the present study should be interpreted carefully. First, the relatively small sample size could not provide sufficient power to elucidate the safety and efficacy of Wuzhi tablet-tacrolimus combination therapy, especially for the impacts of Wuzhi tablet on the other co-administered drugs as well as the long-term outcome of co-administration. Second, this study included only the original twicedaily immediate-release formulation of tacrolimus (Prograft), and therefore the applicability of the co-administration of Wuzhi tablet and other formulations of tacrolimus (such as the once-daily 
prolonged-release formulations) should be further evaluated, since it is well documented that various formulations of tacrolimus are not bioequivalent (Staatz and Tett, 2015).

In conclusion, co-administration of Wuzhi tablet could significantly reduce tacrolimus requirement but without impairing the immunosuppressive effect of tacrolimus and renal allograft function. The tacrolimus dosing regimen guided by CYP3A5 genotype plus Wuzhi tablet co-administration was not only tacrolimus-sparing but also more accurately predicting tacrolimus initial dose for CYP3A5 expressers, suggesting that it is a promising therapy in CYP3A5 expressers in the early post-transplant stage, while further study with a larger sample size is warranted to validate these findings.

\section{Authorship Contributions}

Participated in research design: Li, C. Wang, Huang.

Conducted experiments: Li, Chen, Qin, Fu, Zhang, Liu.

Performed data analysis: Li, Chen, Qin.

Wrote or contributed to the writing of the manuscript: $\mathrm{Li}, \mathrm{Bi}, \mathrm{X}$. Wang.

\section{References}

Aidong W, Zhenjie C, Tong L, Lei Z, Yin W, Shanqi Z, and Liping T (2004) Therapeutic drug monitoring of tacrolimus in early stage after heart transplantation. Transplant Proc 36: 2388-2389.

Chenhsu RY, Loong CC, Chou MH, Lin MF, and Yang WC (2000) Renal allograft dysfunction associated with rifampin-tacrolimus interaction. Ann Pharmacother 34:27-31.

Cornwell MM, Pastan I, and Gottesman MM (1987) Certain calcium channel blockers bind specifically to multidrug-resistant human $\mathrm{KB}$ carcinoma membrane vesicles and inhibit drug binding to P-glycoprotein. J Biol Chem 262:2166-2170.

El-Dahshan KF, Bakr MA, Donia AF, Badr Ael-S, and Sobh MA (2006) Ketoconazole-tacrolimus coadministration in kidney transplant recipients: two-year results of a prospective randomized study. Am J Nephrol 26:293-298.

Fong WF, Wan CK, Zhu GY, Chattopadhyay A, Dey S, Zhao Z, and Shen XL (2007) Schisandrol A from Schisandra chinensis reverses P-glycoprotein-mediated multidrug resistance by affecting Pgp-substrate complexes. Planta Med 73:212-220.

Hebert MF and Lam AY (1999) Diltiazem increases tacrolimus concentrations. Ann Pharmacother 33:680-682.

Hebert MF, Park JM, Chen YL, Akhtar S, and Larson AM (2004) Effects of St. John's wort (Hypericum perforatum) on tacrolimus pharmacokinetics in healthy volunteers. J Clin Pharmacol 44:89-94.

Iwata H, Tezuka Y, Kadota S, Hiratsuka A, and Watabe T (2004) Identification and characterization of potent CYP3A4 inhibitors in Schisandra fruit extract. Drug Metab Dispos 32: $1351-1358$.

Jiang W, Wang X, Xu X, and Kong L (2010) Effect of Schisandra sphenanthera extract on the concentration of tacrolimus in the blood of liver transplant patients. Int J Clin Pharmacol Ther 48:224-229.

Jones DR, Gorski JC, Hamman MA, Mayhew BS, Rider S, and Hall SD (1999) Diltiazem inhibition of cytochrome P-450 3A activity is due to metabolite intermediate complex formation. $J$ Pharmacol Exp Ther 290:1116-1125.

Jones TE and Morris RG (2002) Pharmacokinetic interaction between tacrolimus and diltiazem: dose-response relationship in kidney and liver transplant recipients. Clin Pharmacokinet 41: 381-388.

Jusko WJ, Piekoszewski W, Klintmalm GB, Shaefer MS, Hebert MF, Piergies AA, Lee CC, Schechter P, and Mekki QA (1995) Pharmacokinetics of tacrolimus in liver transplant patients. Clin Pharmacol Ther 57:281-290.

Kidney Disease: Improving Global Outcomes (KDIGO) Transplant Work Group (2009) KDIGO clinical practice guideline for the care of kidney transplant recipients. Am J Transplant 9 (Suppl 3):S1-S155.

Kurzawski M, Dąbrowska J, Dziewanowski K, Domański L, Perużyńska M, and Droździk M (2014) CYP3A5 and CYP3A4, but not ABCB1 polymorphisms affect tacrolimus dose-adjusted trough concentrations in kidney transplant recipients. Pharmacogenomics 15:179-188.

Laskow DA, Vincenti F, Neylan JF, Mendez R, and Matas AJ (1996) An open-label, concentrationranging trial of FK506 in primary kidney transplantation: a report of the United States Multicenter FK506 Kidney Transplant Group. Transplantation 62:900-905.
Li JL, Liu S, Fu Q, Zhang Y, Wang XD, Liu XM, Liu LS, Wang CX, and Huang M (2015) Interactive effects of CYP3A4, CYP3A5, MDR1 and NR1I2 polymorphisms on tracrolimus trough concentrations in early postrenal transplant recipients. Pharmacogenomics 16:1355-1365 Li JL, Wang XD, Chen SY, Liu LS, Fu Q, Chen X, Teng LC, Wang CX, and Huang M (2011) Effects of diltiazem on pharmacokinetics of tacrolimus in relation to CYP3A5 genotype status in renal recipients: from retrospective to prospective. Pharmacogenomics $J$ 11:300-306.

Li JL, Wang XD, Wang CX, Fu Q, Liu LS, Huang M, and Zhou SF (2008) Rapid and simultaneous determination of tacrolimus (FK506) and diltiazem in human whole blood by liquid chromatography-tandem mass spectrometry: application to a clinical drug-drug interaction study. J Chromatogr B Analyt Technol Biomed Life Sci 867:111-118.

Liu C, Shang YF, Zhang XF, Zhang XG, Wang B, Wu Z, Liu XM, Yu L, Ma F, and Lv Y (2009) Co-administration of grapefruit juice increases bioavailability of tacrolimus in liver transplant patients: a prospective study. Eur J Clin Pharmacol 65:881-885.

Loo WT, Cheung MN, and Chow LW (2007) Fructus schisandrae (Wuweizi)-containing compound inhibits secretion of $\mathrm{HBsAg}$ and $\mathrm{HBeAg}$ in hepatocellular carcinoma cell line. Biomed Pharmacother 61:606-610.

Loparev VN, Cartas MA, Monken CE, Velpandi A, and Srinivasan A (1991) An efficient and simple method of DNA extraction from whole blood and cell lines to identify infectious agents. $J$ Virol Methods 34:105-112.

Masuda S and Inui K (2006) An up-date review on individualized dosage adjustment of calcineurin inhibitors in organ transplant patients. Pharmacol Ther 112:184-198.

Montini G, Ujka F, Varagnolo C, Ghio L, Ginevri F, Murer L, Thafam BS, Carasi C, Zacchello G, and Plebani M (2006) The pharmacokinetics and immunosuppressive response of tacrolimus in paediatric renal transplant recipients. Pediatr Nephrol 21:719-724.

Pan Q, Lu Q, Zhang K, and Hu X (2006) Dibenzocyclooctadiene lingnans: a class of novel inhibitors of P-glycoprotein. Cancer Chemother Pharmacol 58:99-106.

Qin XL, Bi HC, Wang CX, Li JL, Wang XD, Liu LS, Chen X, and Huang M (2010a) Study of the effect of Wuzhi tablet (Schisandra sphenanthera extract) on tacrolimus tissue distribution in rat by liquid chromatography tandem mass spectrometry method. Biomed Chromatogr 24:399-405.

Qin XL, Bi HC, Wang XD, Li JL, Wang Y, Xue XP, Chen X, Wang CX, Xu le J, Wang YT, et al. (2010b) Mechanistic understanding of the different effects of Wuzhi tablet (Schisandra sphenanthera extract) on the absorption and first-pass intestinal and hepatic metabolism of Tacrolimus (FK506). Int J Pharm 389:114-121.

Qin XL, Chen X, Wang Y, Xue XP, Wang Y, Li JL, Wang XD, Zhong GP, Wang CX, Yang H, et al. (2014) In vivo to in vitro effects of six bioactive lignans of Wuzhi tablet (Schisandra sphenanthera extract) on the CYP3A/P-glycoprotein-mediated absorption and metabolism of tacrolimus. Drug Metab Dispos 42:193-199.

Qin XL, Yu T, Li LJ, Wang Y, Gu HM, Wang YT, Huang M, and Bi HC (2013) Effect of longterm co-administration of Wuzhi tablet (Schisandra sphenanthera extract) and prednisone on the pharmacokinetics of tacrolimus. Phytomedicine 20:375-379.

Staatz CE and Tett SE (2015) Clinical pharmacokinetics of once-daily tacrolimus in solid-organ transplant patients. Clin Pharmacokinet 54:993-1025.

Venkataramanan R, Swaminathan A, Prasad T, Jain A, Zuckerman S, Warty V, McMichael J, Lever J, Burckart G, and Starzl T (1995) Clinical pharmacokinetics of tacrolimus. Clin Pharmacokinet 29:404-430.

Wan CK, Zhu GY, Shen XL, Chattopadhyay A, Dey S, and Fong WF (2006) Gomisin A alters substrate interaction and reverses P-glycoprotein-mediated multidrug resistance in HepG2-DR cells. Biochem Pharmacol 72:824-837.

Wang K, Qu QS, Zhang YX, Miao SZ, and Jiang X (2016) Effects of Wuzhi capsule on blood concentration of tacrolimus after renal transplantation. J Biol Regul Homeost Agents 30:155-159.

Xin HW, Li Q, Wu XC, He Y, Yu AR, Xiong L, and Xiong Y (2011) Effects of Schisandra sphenanthera extract on the blood concentration of tacrolimus in renal transplant recipients. Eur J Clin Pharmacol 67:1309-1311.

Xin HW, Wu XC, Li Q, Yu AR, Zhu M, Shen Y, Su D, and Xiong L (2007) Effects of Schisandra sphenanthera extract on the pharmacokinetics of tacrolimus in healthy volunteers. Br J Clin Pharmacol 64:469-475.

Xue XP, Qin XL, Xu C, Zhong GP, Wang Y, Huang M, and Bi HC (2013) Effect of Wuzhi tablet (Schisandra sphenanthera extract) on the pharmacokinetics of cyclosporin A in rats. Phytother Res 27:1255-1259.

Zhang Y, Li JL, Fu Q, Wang XD, Liu LS, Wang CX, Xie W, Chen ZJ, Shu WY, and Huang M (2013) Associations of ABCB1, NFKB1, CYP3A, and NR1I2 polymorphisms with cyclosporine trough concentrations in Chinese renal transplant recipients. Acta Pharmacol Sin 34:555-560.

Address correspondence to: Dr. Min Huang, Institute of Clinical Pharmacology, School of Pharmaceutical Sciences, Sun Yat-sen University, 132 Waihuan Dong Road, University City, Guangzhou 510006, People's Republic of China. E-mail: huangmin@mail.sysu.edu.cn; or Dr. Changxi Wang, Department of Organ Transplant, the First Affiliated Hospital of Sun Yat-sen University, 58 Zhongshan Road, Guangzhou 510080, People's Republic of China. E-mail: wcx6363@163.com 